\title{
Carbon Nanotubes-Based Electrochemical Sensors and Drug Delivery Systems: Prospects and Challenges
}

\section{Sandeep Kumar Vashist* and A.G. Venkatesh}

HSG-IMIT-Institut für Mikro- und Informationstechnik, Georges-Koehler-Allee 103, 79110 Freiburg, Germany

Carbon nanotubes (CNTs) are one of the most extensively used nanomaterials in nanomedicine and nanotechnology that has shown tremendous potential for the development of electrochemical sensors (ECS) and drug delivery systems (DDS). Since, the landmark paper by Iijima [1], there have been significant advances in Carbon nanotube based EC sensors (CNT-ECS) and Carbon nanotube based DDS (CNT-DDS), as apparent from the exponentially increasing number of publications and the rapidly growing research interest.

CNTs are hollow cylindrical tubes made up of carbon, having a very high aspect ratio (length/diameter). They have one, two or several concentric graphite layers, capped by fullerenic hemispheres. The single-walled CNTs (SWCNTs) and multi-walled CNTs (MWCNTs) are the most commonly used CNTs (Figure 1), although double-walled CNTs, bamboo CNTs and herringbone have also been developed. The developments in the technology during the last two decades have considerably improved the synthesis and functionalization of CNTs, thereby, resulting in the cost-effective production of CNTs in bulk quantities, and their extensive use for bioanalytical applications. The chemical vapor deposition is the most widely used technique for CNTs synthesis, whereas, arc discharge and laser ablation have also been employed. Various strategies have been devised for the functionalization of CNTs with different chemical groups; conjugation of CNTs to biomolecules; and the preparation of CNT-based electrodes.

CNTs have several unique features, i.e. high mechanical strength, high thermal conductivity and chemical stability, which make them prospective nanomaterial for ECS. The main characteristic of CNTECS is their rapid response with low limit of detection, which is mainly due to their high surface area, low overvoltage and rapid electrode kinetics [2].CNT-ECS have been developed for the detection of glucose, neurotransmitters/neurochemicals, proteins, cells, DNA, microorganisms, pharmaceutical substances and other biomolecules. The direct electron transfer between the enzyme and the CNT-based electrode has further led to the development of mediatorless CNTECS with highly precise analyte detection, as they have negligible interferences from electroactive physiological substances and drug

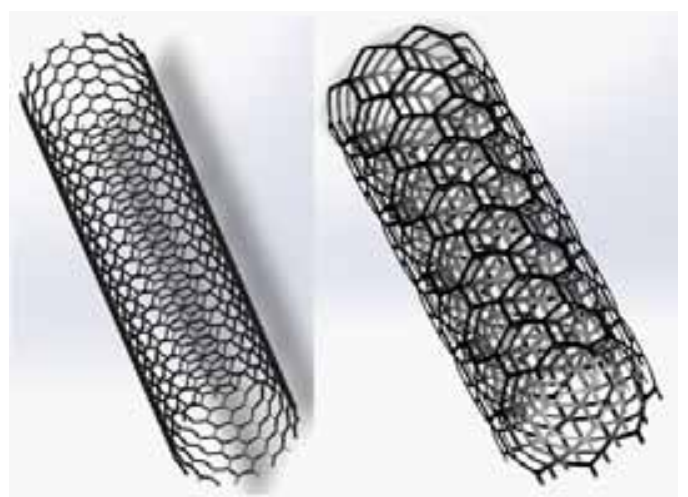

Figure 1: Single-walled (left) and Multi-walled (right) Carbon Nanotubes. metabolites. The chemically-functionalized, vertically-aligned CNTs have also been developed by several researchers and shown to be more sensitive. But, these are highly expensive for the end-users' applications. A wide range of CNT-based nanocomposites and ionic gels have also been developed, to improve the analytical performance of CNT-ECS. CNTs are very promising candidates for the development of next-generation DDS. CNT-DDS enable the delivery of drugs and biomolecules with a very high efficiency due to their large surface area; unique structural, electrical and optical properties; well-defined physico-chemical properties; and no toxicity (using functionalized CNTs). Several conjugation strategies have been developed to covalently/non-covalently bind the drugs/biomolecules to CNTs. CNT-DDS have been extensively employed for the delivery of anticancer, anti-inflammatory and other drugs, in addition to the delivery of biomolecules, i.e. DNA, RNA and proteins [3]. Various formats of CNT-DDS, such as CNT bottles have also been developed [4].

However, despite the significant developments, there are still numerous challenges that need to be tackled before CNT-ECS and CNT-DDS become commercially-viable. The synthesis of ultrapure CNTs without any metallic impurities at a low cost is the main challenge. The pristine CNTs, being intrinsically hydrophobic, cannot disperse uniformly in most solvents and biological media. Therefore, the development of biocompatible CNTs by strategies involving surface modification, surface functionalization or bioconjugation, is critical in order to avoid the toxicity of CNTs. Moreover, the current market price of CNTs is too high and needs to be significantly reduced for realistic commercial applications.

The cytotoxicity of CNTs is affected by many factors such as metal catalyst impurities, length and type of CNTs, and the chemistries being employed for surface functionalization and dispersion of CNTs [5]. Therefore, the determination of the toxicity of CNTs in vitro and in vivo is absolutely essential for any CNT-ECS and CNT-DDS. The absence of international consensus on toxicity analysis at the moment has led to highly conflicting results from various researchers. The drafting of nanotechnology regulation, comprising the international guidelines to determine the toxicity of nanomaterials, is the most challenging task that is currently being chased by the international community [6]. It remains to be seen whether CNTs will conform to the regulatory

*Corresponding author: Sandeep Kumar Vashist, HSG-IMIT-Institut für Mikround Informationstechnik, Georges-Koehler-Allee 103, 79110 Freiburg, Germany, E-mail: sandeep.kumar.vashist@hsg-imit.de

Received October 12, 2012; Accepted October 12, 2012; Published October 15 2012

Citation: Vashist SK, Venkatesh AG (2012) Carbon Nanotubes-Based Electrochemical Sensors and Drug Delivery Systems: Prospects and Challenges. J Nanomed Nanotechol 3:e121. doi:10.4172/2157-7439.1000e121

Copyright: (c) 2012 Vashist SK, et al. This is an open-access article distributed under the terms of the Creative Commons Attribution License, which permits unrestricted use, distribution, and reproduction in any medium, provided the original author and source are credited. 
Citation: Vashist SK, Venkatesh AG (2012) Carbon Nanotubes-Based Electrochemical Sensors and Drug Delivery Systems: Prospects and Challenges. J Nanomed Nanotechol 3:e121. doi:10.4172/2157-7439.1000e121

Page 2 of 2

guidelines and standards of FDA, CE or other regulatory authorities for nanomaterials, and thus get approved for human trials. The actual testing of CNT-ECS and CNT-DDS in the end-user applications' settings, using real-world human samples or humans will then provide the much needed information, pertaining to their analytical performance and interferences.

Although the technology developments in CNT-ECS and CNTDDS are going on at a very fast pace, they are still far from becoming a commercial reality due to the challenges involved. However, the rapid advances in these fields and the evolution of technology will enable researchers to tackle these challenges in near future. There is undoubtedly tremendous technology push for CNT-ECS and CNTDDS due to their numerous advantages over the existing technologies. However, they must comply with the industrial/healthcare requirements before their market entry [7]. The success of commercialization will ultimately be determined by the market pull, generated on the basis of key technology differentiators, i.e. superior analytical performance, cost-effectiveness, robustness, simplicity, signal amplification and reproducibility.

\section{References}

1. lijima S (1991) Helical microtubules of graphitic carbon. Nature 354: 56-58.

2. Vashist SK, Zheng D, Al-Rubeaan K, Luong JHT, Sheu FS (2011) Advances in carbon nanotube based electrochemical sensors for bioanalytical applications. Biotechnol Adv 29: 169-188.

3. Vashist SK, Zheng D, Pastorin G, Al-Rubeaan K, Luong JHT, et al. (2011) Delivery of drugs and biomolecules using carbon nanotubes. Carbon 49: 4077 4097.

4. Li J, Yap SQ, Yoong SL, Nayak TR, Chandra GW, et al. (2012) Carbon nanotube bottles for incorporation, release and enhanced cytotoxic effect of Cisplatin. Carbon 50: 1625-1634.

5. Malloy TF (2011) Nanotechnology regulation: a study in claims making. ACS Nano 5: 5-12.

6. Cui HF, Vashist SK, Al-Rubeaan K, Luong JHT, Sheu FS (2010) Interfacing carbon nanotubes with living mammalian cells and cytotoxicity issues. Chem Res Toxicol 23: 1131-1147.

7. Vashist SK, Venkatesh AG, Mitsakakis K, Czilwik G, Roth G, et al. (2012) Nanotechnology-based biosensors and diagnostics: technology push versus industrial/healthcare requirements. Bionanoscience 2: 115-126. 